\title{
Pacific
}

Journal of

Mathematics

\section{CURRENTS, METRICS AND MOISHEZON MANIFOLDS}

SHANYU JI 


\title{
CURRENTS, METRICS AND MOISHEZON MANIFOLDS
}

\section{SHANYU JI}

\begin{abstract}
A compact complex manifold $M$ is Moishezon if and only if there exists an integral closed positive $(1,1)$-current $\omega$ such that $\omega \geq \varepsilon \sigma$ and $\omega$ is smooth outside an analytic subvariety.
\end{abstract}

1. Introduction. Given a Moishezon manifold $M$, it is well known (cf. [Mo], [W]) that there is a bimeromorphic morphism $\pi: \widetilde{M} \rightarrow M$ such that the manifold $M$ is projective algebraic. Let $\tilde{\omega}$ be Kähler form on $\widetilde{M}$ with $[\tilde{\omega}] \in H^{2}(\widetilde{M}, \mathbf{Z})$. Then the pushforward current $\omega=\pi_{*} \tilde{\omega}$ is a $d$-closed current on $M$ such that

(i) $[\omega] \in H^{2}(M, \mathbf{Z})$;

(ii) $\omega$ is smooth on $M-S$, where $S$ is some proper analytic subset in $M$;

(iii) $\omega \geq \varepsilon \sigma$ in the sense of currents, where $\varepsilon>0$ is some real number and $\sigma$ is a fixed positive definite $(1,1)$-form (not necessarily $d$-closed) on $M$.

Conversely, we shall prove the following

THEOREM 1.1. Let $M$ be a compact complex manifold of dimension $n$. Then $M$ is Moishezon if and only if there exists a d-closed $(1,1)$ current $\omega$ on $M$ such that the conditions (i), (ii) and (iii) above are satisfied.

In fact, the above theorem is a weak version of a general conjecture of Shiffman $[\mathbf{J}]$ which asked: whether a compact complex manifold $N$ is Moishezon if and only if there exists a $d$-closed $(1,1)$-current satisfying the conditions (i) and (iii) above. The conjecture is to generalize the well-known Kodaira embedding theorem in terms of currents and it is still unknown. Some partial results have been obtained [ $\mathbf{J}]$ : if $M$ is complex torus, Shiffman's conjecture is true; if $S$ is a set of isolated points, Theorem 1.1 follows from an extension theorem of Miyaoka [M]; if $S$ is special in some sense, Theorem 1.1 is also true. All of these results are proved by smoothing of currents technique, and depends on a fact that the top degree Chern number $\left(c_{1}([\omega])^{n}, M\right)>0$. However, it is easy to find an example of a current $\omega$ satisfying (i), (ii) 
and (iii) but its top degree Chern number is negative. So the method of smoothing currents cannot prove Theorem 1.1.

Recently, Demailly introduced a very useful notion of singular Hermitian metric on a holomorphic line bundle [D2] and he proved many interesting results. One of them [D2, Proposition 4.2 (b)] is that if $M$ is a projective algebraic manifold of $n$-dimension with a Kähler form $\sigma$ and if $L$ is a holomorphic line bundle over $M$, then $L$ admits a singular Hermitian metric with $c(L) \geq \varepsilon \sigma$ if and only if the Kodaira dimension $\kappa(L)=n$. We observed that this result is in fact the special case of Shiffman's conjecture in which $M$ is projective algebraic (Lemma 2.1). Thus we want to modify Demailly's idea to prove Theorem 1.1. The Demailly's proof is based on the standard $L^{2}$-estimate of $\bar{\partial}$ over Stein or projective algebraic manifolds. However, in our problem, $M$ is only a compact complex manifold. By observing that $M-S$ is complete Kähler (Lemma 4.1), instead of using the standard $L^{2}$-estimate of $\bar{\partial}$, we then prove Theorem 1.1 by using a deep generalization of the $L^{2}$ estimate theorem by Demailly [D1] on complete Kähler manifolds with non-complete Kähler metric and with singular metric on the line bundle. By a similar method, a special case of Shiffman's conjecture when $M$ is Kähler is also proved.

THEOREM 1.2. Let $M$ be a compact Kähler manifold of dimension $n$. Then $M$ is projective algebraic if and only if there exists $a d$ closed $(1,1)$-current $\omega$ on $M$ such that the conditions (i) and (iii) are satisfied.

We also study a class $\mathscr{H}$ of compact complex manifolds as suggested by Harvey and Lawson [HL, $\S 5$, problem 2]. We prove the following result.

TheOREM 1.3. Let $X \in \mathscr{H}$. Then $X$ is a Moishezon manifold iff it is projective algebraic. In particular, this holds for any analytic compact smooth family $X$ of curves with Kähler base space.

Finally we point out an interesting fact below. Its proof is easy from $[\mathbf{K}],[\mathbf{N S}],[\mathbf{N}]$.

THEOREM 1.4. Let $M$ be any compact complex manifold. Then the statements are equivalent:

(i) $M$ is a Moishezon manifold;

(ii) there is a proper analytic subset $S \subset M$ such that $M-S$ admits a complete Kähler-Einstein metric with negative Ricci curvature; 
(iii) There is a proper analytic subset $S \subset M$ such that $M-S$ admits a complete Kähler-Einstein metric with negative Ricci curvature and with finite volume;

(iv) There is a proper analytic subset $S \subset M$ such that $t M-S$ admits a complete Kähler metric $g$ with $\operatorname{Ricci}(g) \leq-g$.

The author wishes to thank Professor B. Shiffman for encouragement for this work and wishes to thank Professor J.-P. Demailly for very stimulating conversation during the JAMI conference at The Johns Hopkins University 1991.

2. Singular metric on line bundles. Let $L$ be a holomorphic line bundle over a complex manifold $M$. A singular Hermitian metric $h$ on $L$ [D2] is a metric which is given in any local trivialization $\theta:\left.L\right|_{U} \rightarrow U \times C$ by

$$
\|\xi\|=|\theta(\xi)| e^{-h_{U}(x)}, \quad x \in U, \xi \in L_{x},
$$

where $h_{U} \in L_{\text {loc }}^{1}(U)$ is an arbitrary function, called the weight of the metric with respect to the trivialization $\theta$. If $\theta^{\prime}:\left.L\right|_{U^{\prime}} \rightarrow U^{\prime} \times \mathbb{C}$ is another trivialization with the associated weight $h_{U}^{\prime}$, and if $\rho \in$ $\mathscr{O}^{*}\left(U \cap U^{\prime}\right)$ is the transition function, then $\theta^{\prime}(\xi)=\rho(x) \theta(\xi)$ for $\xi \in L_{x}$, and $h_{U}^{\prime}=h_{U}+\log |\rho|$ on $U \cap U^{\prime}$. The curvature form of $L$ is then given by the $d$-closed $(1,1)$-current $c(L, h)=\frac{\sqrt{-1}}{\pi} \partial \bar{\partial} h_{U}$ on $U$, which is independent of the choice of local trivialization. The de Rham cohomology class of $c(L, h)$ is the image of the first Chern class $c_{1}(L) \in H^{2}(X, \mathbf{Z})$ in $H_{D R}^{2}(X, \mathbf{R})$.

In order to relate any integral $d$-closed positive $(1,1)$-current to singular Hermitian metric, we need to have the following lemma in the type of Lefschetz' $(1,1)$-theorem.

LEMMA 2.1. Let $M$ be a complex manifold of dimension $n$ and let $\omega$ be a $d$-closed positive $(1,1)$-current on $M$. If the de Rham class $[\omega] \in H^{2}(M, \mathbb{R})$ is integral, then there exists a holomorphic line bundle $L$ with a singular Hermitian metric $h$ such that

$$
\omega=c(L, h) \text {. }
$$

Proof. Choose an open cover $\mathscr{U}=\left\{U_{\alpha}\right\}$ of $M$ such that $U_{\alpha}$ are geometrically convex and then all finite intersections of the sets in $\mathscr{U}$ are contractible. Also assume that each $U_{\alpha}$ is chosen small enough so that there exists a plurisubharmonic function $h_{U_{\alpha}}$ on $U_{\alpha}$ satisfying

$$
\omega=\frac{\sqrt{-1}}{\pi} \partial \bar{\partial} h_{U_{\alpha}}=\frac{1}{2 \pi} d d^{c} h_{U_{\alpha}} \quad \text { on } U_{\alpha}
$$

where $d^{c}=\sqrt{-1}(\bar{\partial}-\partial)$; hence $d d^{c}=2 \sqrt{-1} \partial \sqrt{-1} \partial \bar{\partial}$. 
It is sufficient to find transition functions $\left\{\rho_{\alpha \beta}\right\} \in Z^{1}\left(\mathscr{U}, \mathscr{O}^{*}\right)$ defining a holomorphic line bundle such that

$$
h_{U_{\beta}}=h_{U_{\alpha}}+\log \left|\rho_{\alpha \beta}\right| \text {. }
$$

In this case, $h_{U_{\alpha}}$ is the weight of the singular metric.

Put $u_{j}=\frac{1}{2 \pi} h_{U_{\alpha}}$ and $u_{\alpha \beta}=u_{\beta}-u_{\alpha}$. Because $u_{\beta}-u_{\alpha}$ is pluriharmonic on $U_{\alpha} \cap U_{\beta}$, it implies that $u_{\beta}-u_{j}$ must be smooth; thus $u_{\alpha \beta} \in \mathscr{C}_{\mathbf{R}}^{\infty}\left(U_{\alpha} \cap U_{\beta}\right)$.

By exactly the same argument as in [SS, Lemma 2.36 , p. 38], we can construct a family of transition functions $\left\{\rho_{\alpha \beta}\right\}$ satisfying (2.2). Here we only sketch the proof: since $d d^{c} u_{\alpha \beta}=0$, we can choose $v_{\alpha \beta}=C_{\mathbb{R}}^{\infty}\left(U_{\alpha} \cap U_{\beta}\right)$ such that $d v_{\alpha \beta}=d^{c} u_{\alpha \beta}$. Then $c_{\alpha \beta \gamma}=v_{\beta \gamma}-v_{\alpha \gamma}+$ $v_{\alpha \beta}$ defines an element $\left\{c_{\alpha \beta \gamma}\right\} \in Z^{2}(\mathscr{U}, \mathbf{R})$. By Leray isomorphism, $\left\{c_{\alpha \beta \gamma}\right\}$ corresponds to the cohomology class $[\omega]$. Since $\left\{c_{\alpha \beta \gamma}\right\}$ is integral, there is a 1 -cochain $\left\{b_{\alpha \beta}\right\} \in C^{1}(\mathscr{U}, \mathbf{R})$ such that

$$
c_{\alpha \beta \gamma}+b_{\beta \gamma}-b_{\alpha \gamma}+b_{\alpha \beta}=m_{\alpha \beta \gamma} \in \mathbf{Z} \text {. }
$$

Let $f_{\alpha \beta}=u_{\alpha \beta}+\sqrt{-1}\left(u_{\alpha \beta}+b_{\alpha \beta}\right)$, which is a holomorphic function such that

$$
f_{\beta \gamma}-f_{\alpha \gamma}+f_{\alpha \beta}=\sqrt{-1} m_{\alpha \beta \gamma} .
$$

Let $\rho_{\alpha \beta}=\exp \left(2 \pi f_{\alpha \beta}\right)$. Such $\left\{\rho_{\alpha \beta}\right\} \in Z^{1}\left(\mathscr{U}, \mathscr{O}^{*}\right)$ satisfies (2.2). Thus the lemma is proved.

From the proof above, by the standard regularity theorem for elliptic operators, it is easy to obtain the following

Corollary 2.3. Let $M, \omega$ be as in Lemma 2.1 and let $\omega$ be smooth on $M-S$ for some proper analytic subset $S \subset M$. Then there exists a holomorphic line bundle $L$ over $M$ with a singular metric $h$ such that

$$
\omega=c(L, h)
$$

and $\left.h\right|_{M-S}$ is smooth, i.e., for any point in $x \in M$, there is a neighborhood $U$ of $x$ in $M$ such that the weight $h_{U}$ of the singular metric $h$ is smooth on $U-S$.

Let $L$ be a holomorphic line bundle over $M$ admitting a singular metric $h$ such that the curvature current $c(L, h) \geq 0$. For any $x \in$ $M$, let $h_{U}$ be the weight of the metric on a neighborhood $U$ of $x$, 
we define the Lelong number with respect to the singular metric by (cf. [D2])

$$
v\left(h_{U}, x\right)=\liminf _{z \rightarrow x} \frac{h_{U}(z)}{\log |z-x|}
$$

Equivalently,

$$
v\left(h_{U}, x\right)=\lim _{r \rightarrow 0} v(c(L, h), x, r)
$$

where

$$
v(c(L, h), x, r)=\frac{1}{\left(2 \pi r^{2}\right)^{n-1}} \int_{B(x, r)} c(L, h) \wedge\left(\sqrt{-1} \partial \bar{\partial}|z|^{2}\right)^{n-1} .
$$

So we can denote $v\left(h_{U}, x\right)$ to be $v(c(L, h), x)$. We define a set

$$
E_{c}(c(L, h))=\{x \in M ; v(c(L, h), x) \geq c\}
$$

which is an analytic subset by a well-known theorem of Siu [Si].

LEMMA 2.5 (cf. [D2, Lemma 2.8]). If $\phi$ is a plurisubharmonic function on $M$, then $e^{-2 \phi}$ is integrable in a neighborhood of $x \in M$ if $v(\phi, x)<1$, and $e^{-2 \phi}$ is non-integrable on any neighborhood of $x$ if $v(\phi, x) \geq n$.

3. $L^{2}$ estimate for $\bar{\partial}$ over complete Kähler manifolds. In this section we review some results of Demailly [D1] and state a general $L^{2}$ estimate for $\bar{\partial}$ for line bundles with singular metric.

Let $M$ be a complex manifold of dimension $n$ with a Kähler metric $\omega$. We shall use the same notation $\omega$ to denote the associated Kähler form. Denote $d V_{\omega}=\omega^{n} / n$ ! to be the volume form of $(X, \omega)$. The form $\omega$ defines an operator on $\wedge^{p, q} T^{*} M$ by

$$
\omega(\alpha)=\omega \wedge \alpha \in \bigwedge^{p+1, q+1} T^{*} M
$$

and its adjoint operator $\Lambda$ is defined by

$$
\langle\Lambda \alpha, \beta\rangle=\langle\alpha, \omega(\beta)\rangle
$$

for all $\alpha \in \bigwedge^{p, q} T^{*} M, \beta \in \bigwedge^{p-1, q-1} T^{*} M$. Here $\langle$,$\rangle is the inner$ product given by $\omega$.

Let $L$ be a holomorphic line bundle over $X$. Then these operators $\omega$ and $\Lambda$ can be extended to the space of $L$-valued $(p, q)$-forms, $\bigwedge^{p, q} T^{*} M \otimes L$, by the identity map $\mathrm{id}_{L}$. In additional we suppose that $(L, h)$ is a line bundle over $M$ with a positive $C^{2}$ Hermitian 
metric $h$, i.e., its first Chern class $c_{1}(L, h)>0$. For each integer $q$, $1 \leq q \leq n$, we define a bilinear form $c(L, h)_{q}$

$$
c(L, h)_{q}(\alpha, \beta)=\left\langle 2 \pi c_{1}(L, h) \Lambda \alpha, \beta\right\rangle
$$

for all $\alpha, \beta \in \bigwedge^{n, q} T^{*} M \otimes L$. Since $c_{1}(L, h)>0$, it is known that $c(L, h)_{q}$ is positive, for all $q[\mathrm{D1}$, Lemma 3.1]. For any forms $\alpha \in \bigwedge^{n, q} T^{*} M \otimes L$, one defines

$$
|\alpha|_{c(L, h)_{q}}=\sup _{\beta}\left\{\frac{|\langle\alpha, \beta\rangle|}{c(L, h)_{q}(\beta, \beta)}\right\}
$$

where $0 \neq \beta$ runs through $\bigwedge^{n, q} T^{*} M \otimes L$. Notice that the number $|\alpha|_{c(L, h)_{q}}$ may be equal to infinity. In practice, in order to estimate the term $|\alpha|_{c(L, h)_{q}}$, we have the following result. If $c(L, h) \geq \lambda \omega \otimes$ $\operatorname{Id}_{L}$, where $\lambda \geq 0$ is a measurable function on $M$, then for $\alpha \in$ $\bigwedge^{n, q} T^{*} M \otimes L$, one has [D1, Lemma 3.2]

$$
|\alpha|_{c(L, h)_{q}}^{2} \leq \frac{1}{q \lambda}|\alpha|^{2}
$$

LEMMA 3.2 [D1, Theorem 4.1]. Let $M$ be a complete Kähler manifold of dimension $n$. Let $\omega$ be a Kähler metric which is not necessarily complete. Let $(L, h)$ be a holomorphic Hermitian line bundle over $M$ with a $C^{2}$ positive Hermitian metric $h$. Then for any smooth $L$-valued $(n, q)$-form $g$ on $M$ with

$$
\bar{\partial} g=0, \int_{M}|g|^{2} d V_{\omega}<\infty \quad \text { and } \int_{X}|g|_{c(L, h)_{q}}^{2} d V_{\omega}<\infty,
$$

there exists a smooth L-valued $(n, q-1)$-form $f$ on $M$ such that

$$
\bar{\partial} f=g, \quad \text { and } \quad \int_{M}|f|^{2} d V_{\omega} \leq \int_{X}|g|_{c(L, h)_{q}}^{2} d V_{\omega}
$$

Notice that $M$ is complete Kähler, i.e., $M$ admits a complete Kähler metric $g$, but $\omega$ may not be equal to $g$. The norm || is defined with respect to $\omega$ and $h$.

Let $M$ be a complete Kähler manifold of dimension $n$. Again let $\omega$ be a Kähler metric which is not necessarily complete. Let $L$ be a holomorphic line bundle over $M$ with a $C^{2}$ Hermitian metric $h$. Let $\phi$ be a function on $M$ such that for any point $x \in M$, there is a neighborhood $U$ of $x$ in $M$ such that the restriction of $\phi$ on $U$

$$
\left.\phi\right|_{U}=\phi_{1}+\phi_{2}
$$


where $\phi_{1}$ is a $C^{2}$ function on $U$ and $\phi_{2}$ is a plurisubharmonic function on $U$. The Lebesgue decomposition of the 0 -order current $\sqrt{-1} \partial \bar{\partial} \phi$ gives

$$
\sqrt{-1} \partial \bar{\partial} \phi=\sqrt{-1}(\partial \bar{\partial} \phi)_{c}+\sqrt{-1}(\partial \bar{\partial} \phi)_{s}
$$

where the singular part $\sqrt{-1}(\partial \bar{\partial} \phi)_{s}$ is a positive $(1,1)$-current, and the absolute continuous part $\sqrt{-1}(\partial \bar{\partial} \phi)_{c}$ is a semipositive $(1,1)$ form with $L_{\text {loc }}^{1}$ coefficients.

We define

$$
c\left(L, e^{-\phi} h\right)=c(L, h)+\frac{\sqrt{-1}}{\pi}(\partial \bar{\partial} \phi)_{c} .
$$

LEMMA 3.4 [D1, Theorem 5.1]. Let $M$ be a complete Kähler manifold of dimension $n$. Let $\omega$ be a Kähler metric which is not necessarily complete. Let $L$ be a holomorphic line bundle over $M$ with a $C^{2}$ Hermitian metric $h$. Let $\phi$ be a function which is locally the sum of a $C^{2}$ function and a plurisubharmonic function as in (3.3). Suppose $c\left(L, e^{-\phi} h\right) \geq 0$. Then for any smooth $L$-valued $(n, q)$-form $g$ on $M$ with

$$
\bar{\partial} g=0 \quad \text { and } \quad \int_{M}|g|_{c\left(L, e^{-\phi} h\right)_{q}}^{2} e^{-2 \phi} d V_{\omega}<\infty
$$

there exists a smooth $L$-valued $(n, q-1)$-form $f$ on $M$ such that

$$
\bar{\partial} f=g \quad \text { and } \quad \int_{M}|f|^{2} e^{-2 \phi} d V_{\omega} \leq \int_{M}|g|_{c\left(L, e^{-\phi} h\right)_{q}}^{2} e^{-2 \phi} d V_{\omega} .
$$

where || is defined with respect to $h$ and $\omega$.

The above lemma leads to a general $L^{2}$-estimate for $\bar{\partial}$ for any holomorphic line bundle with singular metric as follows.

Let $M, \omega$ be as in Lemma 3.4 above. Let $L$ be a holomorphic line bundle over $M$ with a singular Hermitian metric $h$. Suppose

$$
c(L, h) \geq 0
$$

in the sense of currents. Now take and fix any smooth Hermitian metric $h_{0}$ and $L$; then on each open subset $U$ such that $\left.L\right|_{U}$ is trivial, the weight $h_{0, U}$ of $h_{0}$ is a smooth function on $U$. Define on each such $U$ a function

$$
\varphi_{U}=h_{U}-h_{0, U} .
$$

It is easy to see that we have in fact defined a function $\varphi$ on $M$ globally such that

$$
\left.\varphi\right|_{U}=\varphi_{U}
$$


Since $c(L, h) \geq 0$, by the proof of Lemma 2.1, we see that any weight $h_{U}$ of the metric $h$ is plurisubharmonic, then the function $\varphi$ is obviously locally a sum of a $C^{2}$-function and a plurisubharmonic function. Then from Lemma 3.4 we obtain

Corollary 3.6. Let $M, \omega$ be as in Lemma 3.4. Let $L$ be a holomorphic line bundle over $M$ with a singular Hermitian metric $h$ such that $c(L, h) \geq 0$. Suppose that $h_{0}$ is any smooth Hermitian metric on $L$ and denote $\varphi$ to be the function on $M$ defined by (3.5). Then for any smooth $L$-valued $(n, q)$-form $g$ on $M$ with

$$
\bar{\partial} g=0 \quad \text { and } \quad \int_{M}|g|_{c\left(L, e^{-\varphi} h_{0}\right)_{q}}^{2} e^{-2 \varphi} d V_{\omega}<\infty,
$$

there exists a smooth L-valued $(n, q-1)$-form $f$ on $M$ such that

$$
\bar{\partial} f=g \quad \text { and } \int_{M}|f|^{2} d V_{\omega} \leq \int_{M}|g|_{c\left(L, e^{-\varphi} h_{0}\right)_{q}}^{2} e^{-2 \varphi} d V_{\omega},
$$

where || is defined by $h$ and $\omega$.

Proof. Apply Lemma 3.4 to $\left(L, h_{0}\right)$ and $\varphi$, we know that for any $g$ with $\bar{\partial} g=0$ and $\int_{M}|g|_{c\left(L, e^{-\varphi} h_{0}\right)_{q}}^{2} e^{-2 \varphi} d V_{\omega}<\infty$, there exists $f$ such that

$$
\bar{\partial} f=g \quad \text { and } \quad \int_{M}|f|_{h_{0}, \omega}^{2} e^{-2 \varphi} d V_{\omega} \leq \int_{M}|g|_{c\left(L, e^{-\varphi} h_{0}\right)_{q}}^{2} e^{-2 \varphi} d V_{\omega} .
$$

Notice that $|f|_{h_{0}, \omega}^{2} e^{-2 \varphi}=|f|_{h, \omega}^{2}$ by (3.5), and the corollary follows.

REMARK 3.7. Suppose that the line bundle $L$ has a singular metric $h$ such that $c(L, h) \geq \varepsilon \omega$, for some constant $\varepsilon>0$, i.e.,

$$
c(L, h)(v, v) \geq \varepsilon \omega(v, v),
$$

for any test form $v$. Since

$$
\begin{aligned}
c\left(L, e^{-\phi} h_{0}\right)(v, v) & =\left[c\left(L, h_{0}\right)+\left(\frac{\sqrt{-1}}{\pi} \partial \bar{\partial}\right)_{c}\right](v, v) \\
& =\left[c\left(L, h_{0}\right)+\frac{\sqrt{-1}}{\pi} \partial \bar{\partial} \phi\right](v, v) \\
& =c(L, h)(v, v),
\end{aligned}
$$

we see that

$$
c\left(L, e^{-\phi} h_{0}\right)(v, v) \geq \varepsilon \omega(v, v)
$$


Therefore by (3.1) we have the estimate

$$
|\alpha|_{c\left(L, e^{-\phi} h_{0}\right)_{q}}^{2} \leq \text { const } .|\alpha|^{2}
$$

for any $\alpha \in \bigwedge^{n, q} T^{*} M \otimes L$.

4. Complete Kähler metric on $M-S$. In order to prove Theorem 1.1 , we wish to apply Lemma 3.4 to $X-S$. In general, $X-S$ may not be complete Kähler, but together with the $d$-closed $(1,1)$-current $\omega$ satisfying (ii) and (iii), we can construct a complete Kähler metric on $M-S$. The proof is analogous to [D1, Proposition 1.6].

LEMMA 4.1. Let $M$ be a compact complex manifold and $S \subset M a$ proper analytic subset. Let $\omega$ be a d-closed $(1,1)$-current satisfying the conditions (ii) and (iii) in $\S 1$. Then $M-S$ admits a complete Kähler metric.

Proof. By [D1, Proposition 1.4], for any complex manifold $M$ and any analytic subset $S \subset M$, there exists a locally integrable function $\psi$ on $M$ such that $\psi$ is smooth on $M-S ; \psi(x)<-1$, for any $x \in M-S$; and $\psi(x) \rightarrow-\infty$ as $x$ goes to $S$, and there exists a real continuous $(1,1)$-form $\gamma$ on $X$ such that

(4.2) $\sqrt{-1} \partial \bar{\partial} \psi \geq \gamma$

(4.3) if $\alpha>0$ is a real number, $e^{-\alpha \psi}$ is non-integrable on a neighborhood of a point $s \in S$ where the codimension of the germ $S_{s}$ satisfies

$$
\operatorname{codim} S_{s} \geq \alpha .
$$

Put $\tilde{\omega}=C \omega-\sqrt{-1} \partial \bar{\partial} \sqrt{-\psi}$, which is a smooth form on $M-S$ and is a current on $M$. We claim that we can choose the constant $C>0$ large enough such that

$$
(C-1) \omega+\frac{\sqrt{-1} \partial \bar{\partial} \psi}{2 \sqrt{-\psi}} \geq 0 \quad \text { on } M-S .
$$

In fact, since $\gamma$ is continuous on $M$ and $\omega \geq \varepsilon \sigma$ on $M$ (cf. (iii) in $\S 1$, and since $X$ is compact, we can find a constant number $C>0$ such that

$$
(C-1) \varepsilon \sigma \geq-\frac{\gamma}{2 \sqrt{-\psi}} \text { on } M
$$

Then

$$
(C-1) \omega+\frac{\sqrt{-1} \partial \bar{\partial} \psi}{2 \sqrt{-\psi}} \geq(C-1) \varepsilon \sigma+\frac{\gamma}{2 \sqrt{-\psi}} \geq 0
$$


on $M$ in the sense of currents. Thus (4.4) is proved. From (4.4), it yields

$$
\tilde{\omega} \geq \omega+4 \sqrt{-1} \partial(-\psi)^{1 / 4} \wedge \bar{\partial}(-\psi)^{1 / 4}
$$

because

$$
\sqrt{-1} \partial \bar{\partial}(-\sqrt{-\psi})=\frac{\sqrt{-1} \partial \bar{\partial} \psi}{2 \sqrt{-\psi}}+4 \sqrt{-1} \partial(-\psi)^{1 / 4} \wedge \bar{\partial}(-\psi)^{1 / 4} .
$$

Then $\tilde{\omega}$ is complete Kähler by the same argument of [D1, proof of Proposition 1.6]. For the reader's convenience, we still give the proof: let $\delta$ (resp. $\tilde{\delta})$ be the geodesic distance associated to $\omega$ (resp. $\tilde{\omega})$. For any two $z_{1}, z_{2} \in M$,

$$
\delta\left(z_{1}, z_{2}\right)=\inf \int_{0}^{1} \sqrt{\omega\left(\frac{d u}{d t}, \sqrt{-1} \frac{d u}{d t}\right)} d t
$$

(similarly one defines $\tilde{\delta}\left(z_{1}, z_{2}\right)$ ) where $u$ runs through the set of all $C^{1}$ curves $u:[0,1] \rightarrow M-S$ with the ending points $z_{1}$ and $z_{2}$. By (4.5),

$$
\begin{aligned}
\tilde{\omega}\left(\frac{d u}{d t}, \sqrt{-1} \frac{d u}{d t}\right) & \geq \omega\left(\frac{d u}{d t}, \sqrt{-1} \frac{d u}{d t}\right)+4\left|\partial \psi\left(\frac{d u}{d t}\right)\right|^{2} \\
& \geq \omega\left(\frac{d u}{d t}, \sqrt{-1} \frac{d u}{d t}\right)+\left|\frac{d(\psi \circ u)}{d t}\right|^{2}
\end{aligned}
$$

because

$$
\frac{d(\psi \circ u)}{d t}=d \psi\left(\frac{d u}{d t}\right)=2 \operatorname{Re} \partial \psi\left(\frac{d u}{d t}\right) .
$$

Thus

$$
\tilde{\delta}\left(z_{1}, z_{2}\right) \geq \sup \left(\delta\left(z_{1}, z_{2}\right),\left|\psi\left(z_{1}\right)-\psi\left(z_{2}\right)\right|\right) .
$$

Since $\psi$ is exhaustive, and since a manifold admits a complete metric $\tilde{\omega}$ if and only if the closed balls defined by geodesic distance $\tilde{\delta}$ are always compact, we know that $\tilde{\omega}$ is complete Kähler on $M-S$.

\section{Proofs of Theorems 1.1 and 1.2.}

LEMMA 5.1 [D1, Lemma 6.9]. Let $\Omega \subset \mathbf{C}^{n}$ be an open subset, and let $Y \subset \Omega$ be an analytic subset. If $w$ is $a(p, q)$-form with $L_{\text {loc }}^{1}$ coefficients on $\Omega$, and $v$ is $a(p, q-1)$-form with $L_{\text {loc }}^{2}$ coefficients on $\Omega$ such that $\bar{\partial} v=w$ on $\Omega-Y$ in the sense of currents, then $\bar{\partial} v=w$ on $\Omega$ in the sense of currents.

Proof of Theorem 1.1. By Lemma 2.1 and Corollary 2.2, there exists a holomorphic line bundle $L$ over $M$ with a singular metric $h$ such that $\omega=c(L, h)$ and $h$ is smooth on $U-S$. 
By Lemma 4.1, $M-S$ is a complete Kähler manifold. Since the restriction of the singular metric $h$ on $L$ is smooth over $M-S$, $\left.L\right|_{M-S}$ has a smooth Hermitian $h=\left.h\right|_{M-S}$. We consider $\omega$ as Kähler metric on $M-S$. Notice that $\omega$ is not necessarily complete.

Take and fix a point $x_{0} \in M-S$. Because $h$ is smooth on $M-S$, we see that the Lelong number is

$$
v(c(L, h), x)=0, \quad x=x_{0} \text { or } x \text { near } x_{0} .
$$

Let $\Psi_{0}$ be a smooth function on $M-\left\{x_{0}\right\}$ which is equal to $n \log \left|z-x_{0}\right|$ (in some coordinates) near $x_{0}$.

By the hypothesis (iii), $c(L, h) \geq \varepsilon \sigma$ on $M$, there is some $m$ such that

$$
m c(L, h)+\frac{\sqrt{-1}}{\pi} \partial \bar{\partial} \Psi_{0} \geq m \varepsilon \sigma+\frac{\sqrt{-1}}{\pi} \partial \bar{\partial} \Psi_{0} \geq \sigma .
$$

Put $v_{m}=c(L, h)+(\sqrt{-1} / \pi) \partial \bar{\partial} \Psi_{0}$. The Lelong number

$v\left(v_{m}, x_{0}\right)=n+1 \quad$ and $\quad v\left(v_{m}, z\right)<1, \quad$ for $z \neq x_{0}$ near $x_{0}$.

Therefore, by Lemma $2.5, e^{-2 \Psi_{0}}$ is non-integrable near $x_{0}$.

Let $P(z)$ be an arbitrary polynomial of degree 1 in the given coordinates $V$ of $x_{0}$. Fix a smooth cut-off function $\chi$ with compact support in $V$ such that $\chi=1$ near $x_{0}$. Fix a non-vanishing local holomorphic section $g \in H^{0}\left(V, K_{M} \otimes L^{m}\right)$.

Then $v=P \bar{\partial} \chi \otimes g$ is regarded as a smooth $\bar{\partial}$-closed $L^{m}$-valued $(n, 1)$-form on $M$ and hence on $M-S$ such that

$$
\int_{M-S}|v|^{2} e^{-2 \Psi_{0}} d V_{\omega}<\infty
$$

where $\Psi_{0}$ is constructed as above, and || is defined by $\omega$ and by the smooth Hermitian metric $h$.

Then by (5.2), we apply Lemma 3.4 and Remark 3.7 to $M-S$, $\left(L^{m}, h^{m}\right)$ and $\Psi_{0}$, and then there is a smooth $L^{m}$-valued $(n, 0)$ form $u$ on $M-S$ such that

$$
\bar{\partial} u=v \quad \text { and } \quad \int_{M-S}|u|^{2} e^{-2 \Psi_{0}} d V_{\omega} \leq \int_{M-S}|v|^{2} e^{-2 \Psi_{0}} d V_{\omega}<\infty .
$$

Then we claim that $u$ can be extended as a smooth $L^{m}$-valued $(n, 0)$-form on $M$. In fact, we apply Lemma 5.1 to prove it. Since $v$ is a smooth $L^{m}$-valued $(n, 0)$-form on $M$, it is sufficient to show that $\forall x \in S$, there is a neighborhood $U=U_{x}$ of $x$ in $M$ such that

$$
\int_{U-S}|u|_{U}^{2} d V_{U}<\infty
$$


where $d V_{U}$ is the Euclidean volume form on $U$ with respect to a coordinate system and the norm $\|_{U}$ is with respect to $d V_{U}$ and $h$. Recall $\omega \geq \varepsilon \sigma$, and $\Psi_{0}$ is smooth near $S$; it yields

$$
\int_{U}|u|_{U}^{2} d V_{U}=\int_{U-S}|u|_{U}^{2} d V_{U} \leq \text { constant } \int_{U-S}|u|^{2} e^{-2 \Psi_{0}} d V_{\omega}<\infty .
$$

The claim then is proved by the regularity theorem.

Also we claim that $|u(z)|=o\left(\left|z-x_{0}\right|\right)$ near $x_{0}$. In fact, it is true by the fact that $\int_{M-S}|u|^{2} e^{-2 \Psi_{0}} d V<\infty$, and that $e^{-2 \Psi_{0}}$ is nonintegrable near $x_{0}$, and that $u$ is holomorphic near $x_{0}$.

Therefore

$$
f:=\chi P g-u \in H^{0}\left(M, K_{M} \otimes L^{m}\right)
$$

has the prescribed 1-jet $P g$ at $x_{0}$. Thus the Kodaira dimension of $K_{M} \otimes L^{m}=n$. Hence $M$ is Moishezon.

Proof of Theorem 1.2. By the similar procedure as above, replacing $M-S$ by $M$, we can apply Corollary 3.6 and Remark 3.7 to know that $M$ is Moishezon if and only if there is a $(1,1)$-form $\omega$ satisfying (i) and (iii). Since $M$ is Kähler, by Moishezon's theorem [Mo], it is equivalent to $M$ being projective algebraic. Then Theorem 1.2 follows.

6. Projectivity of a class of Moishezon manifolds. In 1983, Harvey and Lawson proved a characterization theorem for Kähler manifolds [HL], that is, a compact complex manifold is Kähler if and only if there exists no nontrivial positive current which is a bidimension $(1,1)$-component of a boundary. They also raised several general problems. One of them [HL, $\S 5$, problem 2] is as follows: describe the class of compact complex manifolds which satisfy that if there exists a non-trivial positive current which is the bidimension $(1,1)$-component of a boundary, then there exists a non-trivial positive smooth current which is the bidimension $(1,1)$-component of a boundary. We denote this class by $\mathscr{H}$. The significance of this problem is that to test whether a given manifold in $\mathscr{H}$ is Kähler; it suffices to check the pointwise non-negative, smooth $(n-1, n-1)$-forms, to see if one is a boundary.

It is worth remarking that investigating the obstruction of a Moishezon manifold to be projective algebraic is an interesting problem in the theory of compact complex manifolds. Classically we know that there is no obstruction for compact complex surfaces (Chow-Kodaira [CK]) and for complex tori (Lefschetz [W]). Moishezon's theorem 
[Mo] just means that this obstruction is equivalent to that the manifold is non-Kähler. Recently Peternell showed that this obstruction for 3-dimensional complex manifolds is a positive integral linear combination of irreducible curves which is homologous to zero.

As an important example in $\mathscr{H}$, we point out that if $(X, Y, f)$ is any analytic compact smooth family of curves with Kähler base space $Y$, then $X$ is in $\mathscr{H}$. Here we say that $(X, Y, f)$ is an analytic compact smooth family of curves if $X$ and $Y$ are compact connected complex manifolds, and $f: X \rightarrow Y$ is a surjective holomorphic map which is everywhere of maximal rank such that each fiber $X_{y}=f^{-1}(y)$ is a connected smooth curve for any $y \in Y$. Notice that in this case $f$ is a submersion. For any analytic compact smooth family of curves $(X, Y, f)$ with Kähler base space $Y$, we know $X \in \mathscr{H}$ by [HL, Theorem $(17)^{\infty}$ ].

Proof of the Theorem 1.3. By applying Moishezon's theorem [Mo] that a Moishezon manifold is projective algebraic if and only if it is Kähler, it suffices to show: if $X$ is a Moishezon manifold, then $X$ is a Kähler manifold. Suppose $X$ is non-Kähler. By the result of Harvey and Lawson [HL, Proposition (12) and Theorem (14)], we know that there exists a non-trivial positive current $T_{0}$ on $X$ which is the bidimension $(1,1)$-component of a boundary. Since $X \in \mathscr{H}$, there exists a non-trivial positive smooth current $T$ which is the bidimension (1,1)-component of a boundary. We can write

$$
T=\partial S^{2,1}+\bar{\partial} S^{1,2}
$$

where $S^{2,1}$ and $S^{1,2}$ are some currents of $X$ of the bidimension $(2,1)$ and $(1,2)$, respectively. Notice that these $S^{2,1}$ and $S^{1,2}$ may not be smooth.

Since $X$ is Moishezon, there exists a modification $\pi: \widetilde{X} \rightarrow X$ such that the manifold $\widetilde{X}$ is projective algebraic. Let $\tilde{\sigma}$ be a Kähler form on $\widetilde{X}$.

We claim that the push-forward current $\sigma:=\pi_{*} \tilde{\sigma}$ is a $d$-closed $(1,1)$-current on $X$ satisfying the following property: for any point $a \in X$, there exists a neighborhood $U$ of $a$ in $X$ with a local coordinates system $\left(U, z^{1}, \ldots, z^{n}\right)$ and a positive constant $C$ such that

$$
\sigma-C \sum_{j=1}^{n} \sqrt{-1} d z^{j} \wedge d \bar{z}^{j} \geq 0
$$


on $U$ in the sense of currents. In fact, for any point $a \in M$, we can find a neighborhood $U$ of $a$ in $M$ with a local coordinate system $\left(U, z^{1}, \ldots, z^{n}\right)$ and a constant $C>0$ such that

$$
\tilde{\sigma}-C \pi^{*}\left(\sum_{j=1}^{n} \sqrt{-1} d z^{j} \wedge d \bar{z}^{j}\right) \geq 0
$$

on $\pi^{-1}(U)$ in the sense of currents. Then on $U$, we see $\pi_{*} \tilde{\sigma}-$ $C \pi_{*} \pi^{*}\left(\sum_{j=1}^{n} \sqrt{-1} d z^{j} \wedge d \bar{z}^{j}\right) \geq 0$ in the sense of currents. So we have $\sigma=\pi_{*} \tilde{\sigma} \geq C \pi_{*} \pi^{*}\left(\sum_{j=1}^{n} \sqrt{-1} d z^{j} \wedge d \bar{z}^{j}\right) \geq C \sum_{j=1}^{n} \sqrt{-1} d z^{j} \wedge d \bar{z}^{j}$ on $U$ in the sense of currents. The claim (6.1) is then proved.

We define the smoothing $\sigma_{\varepsilon}$ as follows: Let $\left\{U_{i}\right\}_{1 \leq i \leq}$ be any finite open covering of $X$ and $\left\{\varphi_{i}\right\}_{1 \leq i \leq q}$ be any partition of unity subordinate to $\left\{U_{i}\right\}_{1 \leq i \leq q}$. Suppose that every $U_{i}$ is a coordinate chart and that $U_{i}$ is identified with a unit ball with center $0 \in \mathbf{C}^{n}$ with respect to the coordinate chart. On each $U_{i}$, since it is biholomorphic to the open unit ball, we can write

$$
\sigma=\sqrt{-1} \partial \bar{\partial} f_{i}
$$

Because $f_{i}-f_{j}$ is pluriharmonic on $U_{i} \cap U_{j}, \forall i \neq j$, it implies that $f_{j}=f_{i}$ is smooth. Then we define a global $d$-closed smooth real $(1,1)$-form $P$ on $X$

$$
P:=\omega-\sqrt{-1} \partial \bar{\partial} \sum_{i=1}^{r} \varphi_{i} f_{i}
$$

because $\left.P\right|_{U_{j}}=\sqrt{-1} \partial \bar{\partial} \sum_{i=1}^{r} \varphi_{i}\left(h_{j}-h_{i}\right)$; i.e., we have $\sigma=P+$ $\sqrt{-1} \sum_{i} \varphi_{i} f_{i}$. Then smoothing $\sigma_{\varepsilon}$ of $\sigma$, a $d$-closed real $(1,1)$-form on $X$, is defined by

$$
\sigma_{\varepsilon}=P+\sqrt{-1} \partial \bar{\partial}\left(\sum_{i=1}^{q} \chi_{i, \varepsilon} *\left(\varphi_{i} f_{i}\right)\right),
$$

where $\chi_{i, \varepsilon}$ is the standard approximation of identity defined on $U_{i}$.

Since $\sigma_{\varepsilon}-\sigma=\sqrt{-1} \partial \bar{\partial}\left\{\sum_{i=1}^{q}\left(\chi_{i, \varepsilon} *\left(\varphi_{i} f_{i}\right)-\left(\varphi_{i} f_{i}\right)\right)\right\}$, it follows that

$$
[\sigma]=\left[\sigma_{\varepsilon}\right] \in H^{1,1}(X, \mathbf{R}) \quad \text { and } \quad \sigma_{\varepsilon} \rightarrow \sigma \text {, as } \varepsilon \rightarrow 0
$$

in the sense of currents.

By the facts that $X$ is compact and that $T$ is smooth, we can make the following computation:

$$
\begin{aligned}
(\sigma, T) & =\lim _{\varepsilon \rightarrow 0}\left(\sigma_{\varepsilon}, T\right)=\lim _{\varepsilon \rightarrow 0}\left(\sigma_{\varepsilon}, \partial S^{2,1}+\bar{\partial} S^{1,2}\right) \\
& =\lim _{\varepsilon \rightarrow 0}(0+0)=0,
\end{aligned}
$$

where we used the fact that $\sigma_{\varepsilon}$ is $d$-closed. 
On the other hand, the following claim leads to a contradiction and it completes the proof of the theorem:

$$
(\sigma, T)>0 .
$$

In fact, let the open covering $\left\{U_{i}\right\}_{1 \leq i \leq q}$ of $X$ and a partition of unity $\left\{\varphi_{i}\right\}_{1 \leq i \leq q}$ subordinate to $\left\{U_{i}\right\}_{1 \leq i \leq q}$ be as before. Since $T$ is non-trivial and $\sigma$ satisfies the property $(6.1)$, we assume that the form $\varphi_{1} T \neq 0$ and that $\sigma \geq C \sum_{j=1}^{n} \sqrt{-1} d z_{j} \wedge d \bar{z}_{j}$ on $U_{1}$ for some positive constant number $C$ in the sense of currents. Then

$$
(\sigma, T)=\sum_{i=1}^{q}\left(\sigma, \varphi_{i} T\right) .
$$

We claim that for each $i, 1 \leq i \leq q,\left(\sigma, \varphi_{i} T\right) \geq 0$. In fact, let $\chi_{i, \varepsilon}$ be the standard approximation of identity defined on $U_{i}$. Then $\left(\sigma, \varphi_{i} T\right)=\lim _{\varepsilon \rightarrow 0}\left(\chi_{i, \varepsilon} * \sigma, \varphi_{i} T\right)$. Notice that $\chi_{i, \varepsilon} * \sigma$ is a positive $C^{\infty}(1,1)$-form on $U_{i}$ for any $\varepsilon>0$, and that $\varphi_{i} T$ is positive $(n-1, n-1)$-current on $U_{i}$; it follows that $\left(\chi_{i, \varepsilon} * \sigma, \varphi_{i} T\right)$ is nonnegative. The claim then is verified by letting $\varepsilon$ go to zero. Therefore, we have shown

$$
(\sigma, T) \geq\left(\sigma, \varphi_{1} T\right) .
$$

For the positive current $\sigma-C \sum_{j=1}^{n} \sqrt{-1} d z_{j} \wedge d \overline{z_{j}}$ on $U_{1}$, by applying the same method, we know $\left(\sigma-C \sum_{j=1}^{n} \sqrt{-1} d z_{j} \wedge d \overline{z_{j}}, \varphi_{1} T\right) \geq 0$, i.e.,

$$
\left(\sigma, \varphi_{1} T\right) \geq C\left(\sum_{j=1}^{n} \sqrt{-1} d z_{j} \wedge d \overline{z_{j}}, \varphi_{1} T\right) .
$$

Applying Wirtinger's Inequality as well as the argument in [HL, $\S 4]$, we get

$$
\left(\sum_{j=1}^{n} \sqrt{-1} d z_{j} \wedge d \overline{z_{j}}, \varphi_{1} T\right)=M\left(\varphi_{1} T\right)>0,
$$

where $M\left(\varphi_{1} T\right)$ is the mass of $T$. The claim (6.2) follows from (6.3), (6.4), (6.5) above.

7. Moishezon manifolds and Kähler-Einstein metrics. We prove Theorem 1.4 now. The statements (ii) $\Rightarrow$ (ii) $\Rightarrow$ (iv) are trivial. It suffices to show (i) $\Rightarrow$ (iii) and (iv) $\Rightarrow(\mathrm{v})$. 
Proof for (i) $\Rightarrow$ (iii). Suppose that $M$ is a Moishezon manifold. Then there is a projective algebraic manifold $\widetilde{M}$, a proper surjective holomorphic mapping $\pi: \widetilde{M} \rightarrow M$, and an analytic set $V$ such that the restriction mapping $\left.\pi\right|_{\widetilde{M}-\widetilde{V}}: \widetilde{M}-\widetilde{V} \rightarrow M-V$ is bihomororphic, where $\widetilde{V}:=\pi^{-1}(V)$. Choose a purely 1-codimensional analytic subset $\widetilde{S}$ on $\widetilde{M}$ such that $\widetilde{V} \subset \widetilde{S}$ and that $K_{M} \otimes[\widetilde{S}]$ is ample. By applying Hironaka's resolution of singularities if necessary, we assume without loss of generality that $\widetilde{S}$ is with simple normal crossings. Then by a result of Kobayashi [K, Theorem 1], we know that $\widetilde{M}-\tilde{S}$ admits a complete Kähler-Einstein metric $\tilde{g}$ which is with negative Ricci curvature and with finite volume. Then we set $g:=\left(\left(\left.\pi\right|_{M-S}\right)^{-1}\right)^{*} \tilde{g}$.

Proof for (iv) $\Rightarrow$ (i). Suppose that there is an analytic subset $S \subset M$ such that $M-S$ admits a complete Kähler metric $g$ with Ricci $(g) \leq$ $-g$. By Hironaka's resolution of singularities again if necessary, we can assume $S$ is a hypersurface. Then by the $L^{2}$ Riemann-Roch inequality proved by Nadel and Tsuji [NS] and by [N, Proposition 1.11], it implies

$$
\begin{aligned}
\liminf _{k \rightarrow+\infty} & \frac{1}{k^{n}} \operatorname{dim} H^{0}\left(M, K_{K}^{\otimes k} \times[S]^{\otimes(k-1)}\right) \\
& \geq \liminf _{k \rightarrow+\infty} \frac{1}{k^{n}} \operatorname{dim} H_{(2)}^{0}\left(j, K_{M-S}^{\otimes k}\right) \\
& \geq \frac{1}{n !} \int_{M-S} c_{1}\left(K_{M-S}\right)^{n} \geq \frac{1}{n !} \int_{M-S} g^{n}>0 .
\end{aligned}
$$

Since $H^{0}\left(M, K_{M}^{\otimes k} \otimes[S]^{\otimes(k-1)}\right) \subset H^{0}\left(M,\left(K_{M} \otimes[S]\right)^{k}\right)$, we then see

$$
\begin{aligned}
& \operatorname{dim} H^{0}\left(M,\left(K_{M} \otimes[S]\right)^{\otimes k}\right) \\
& \quad \geq \frac{k^{n}}{n !} \int_{M-S} g^{n}+O\left(k^{n}\right)=\left(\frac{1}{n !} \int_{M-S} g^{n}+\frac{O\left(k^{m}\right)}{k^{n}}\right) k^{n} .
\end{aligned}
$$

This yields (i).

Note added in proof. Recently, Shiffman's conjecture has been proved completely. See: S. Ji and B. Shiffman, Properties of compact complex manifolds carrying closed positive currents, to appear in J. Geom. Anal. 


\section{REFERENCES}

[CK] W. L. Chow and K. Kodaira, On analytic surfaces with two independent meromorphic functions, Proc. Nat. Acad. Sci. U.S.A., 38 (1952), 319-325.

[D1] J.-P. Demailly, Estimations $L^{2}$ pour l'opérateur $\bar{\partial}$ d'un fibré vectoriel holomophe semi-positif au dessus d'une variété kählérienne complète, Ann. Sci. Ec. Norm. Sup., 15 (1982), 457-511.

[D2] - Singular Hermitian metrics on positive line bundles, Proceedings of the conference "Complex Algebraic Varieties", Lecture Notes in Math., vol. 1507, Springer-Verlag, (1992), 87-104.

[HK] R. Harvey and A. W. Knapp, Positive $(p, p)$-forms, Wirtinger's inequality, and currents, Proc. of the Tulane Conference on Value Distribution theory, Part A, Marcel Dekker, New York (1974), 43-62.

[HL] R. Harvey and H. B. Lawson, Jr., An intrinsic characterization of Kähler manifolds, Invent. Math., 74 (1983), 169-198.

[K] R. Kobayashi, Kähler-Einstein metric on an open algebraic manifold, Osaka J. Math., 21 (1984), 399-418.

[J] S. Ji, Smoothing of currents and Moisezon manifolds, Proceedings of Symposia in Pure Math., vol. 52 (1991), Part 2, 273-282.

[M] Y. Miyaoka, Extension theorem for Kahler metrics, Proc. Japan Acad., 50 (1974), 407-409.

[Mo] B. G. Moišezon, On n-dimensional compact varieties with $n$ algebraically independent meromorphic functions, Amer. Math. Soc. Transl., 63 (1967), 51 177.

[NS] A. Nadel and H. Tsuji, Compactification of complete Kähler manifolds of negative Ricci curvature, J. Differential Geom., 28 (1988), 503-512.

[N] A. Nadel, On complex manifolds which can be compactified by adding finitely many points, Invent. Math., 101 (1990), 173-189.

[Si] Y.-T. Siu, Analyticity of sets associated to Lelong numbers and the extension of closed positive currents, Invent. Math., 27 (1974), 53-156.

[SS] B. Shiffman and A. J. Sommese, Vanishing Theorems on Complex Manifolds, Birkhäuser, Boston-Basel-Stuttgart, 1985.

[W] R. O. Wells, Moišezon spaces and the Kodaira embedding theorem, Value Distribution Theory, Part A, Marcel Dekker, New York, 1974, 29-42.

Received June 27, 1991. Partial financial support was provided by the NSF under grant number DMS-8922760 and by a Research Initiation Grant at University of Houston in 1990.

UNIVERSITY OF HOUSTON

Houston, TX 77204 



\title{
PACIFIC JOURNAL OF MATHEMATICS \\ Founded by \\ E. F. BECKENBACH (1906-1982) F. WolF (1904-1989)
}

\section{EDITORS}

\section{S. VARADARAJAN \\ (Managing Editor) \\ University of California \\ Los Angeles, CA 90024-1555 \\ vsv@math.ucla.edu}

\section{F. Michael Christ}

University of California

Los Angeles, CA 90024-1555

christ@math.ucla.edu

\section{Herbert Clemens}

University of Utah

Salt Lake City, UT 84112

clemens@math.utah.edu

\author{
THOMAs ENRIGHT \\ University of California, San Diego \\ La Jolla, CA 92093 \\ tenright@ucsd.edu \\ Nicholas ERcolani \\ University of Arizona \\ Tucson, AZ 85721 \\ ercolani@math.arizona.edu \\ R. FINN \\ Stanford University \\ Stanford, CA 94305 \\ finn@gauss.stanford.edu \\ VAUghan F. R. JoNeS \\ University of California \\ Berkeley, CA 94720 \\ vfr@math.berkeley.edu
}

\section{SUPPORTING INSTITUTIONS}

UNIVERSITY OF ARIZONA

UNIVERSITY OF BRITISH COLUMBIA

CALIFORNIA INSTITUTE OF TECHNOLOGY

UNIVERSITY OF CALIFORNIA

UNIVERSITY OF MONTANA

UNIVERSITY OF NEVADA, RENO

NEW MEXICO STATE UNIVERSITY OREGON STATE UNIVERSITY

\author{
UNIVERSITY OF OREGON \\ UNIVERSITY OF SOUTHERN CALIFORNIA \\ STANFORD UNIVERSITY \\ UNIVERSITY OF HAWAII \\ UNIVERSITY OF UTAH \\ WASHINGTON STATE UNIVERSITY \\ UNIVERSITY OF WASHINGTON
}

The Supporting Institutions listed above contribute to the cost of publication of this Journal, but they are not owners or publishers and have no responsibility for its content or policies.

Mathematical papers intended for publication in the Pacific Journal of Mathematics should be in typed form or offset-reproduced (not dittoed), double spaced with large margins. Please do not use built up fractions in the text of the manuscript. However, you may use them in the displayed equations. Underline Greek letters in red, German in green, and script in blue. The first paragraph must be capable of being used separately as a synopsis of the entire paper. In particular it should contain no bibliographic references. Please propose a heading for the odd numbered pages of less than 35 characters. Manuscripts, in triplicate, may be sent to any one of the editors. Please classify according to the 1991 Mathematics Subject Classification scheme which can be found in the December index volumes of Mathematical Reviews. Supply name and address of author to whom proofs should be sent. All other communications should be addressed to the managing editor, or Julie Speckart, University of California, Los Angeles, California 90024-1555.

There are page-charges associated with articles appearing in the Pacific Journal of Mathematics. These charges are expected to be paid by the author's University, Government Agency or Company. If the author or authors do not have access to such Institutional support these charges are waived. Single authors will receive 75 free reprints; joint authors will receive a total of 100 free reprints. Additional copies may be obtained at cost in multiples of 50 .

The Pacific Journal of Mathematics (ISSN 0030-8730) is published monthly except for July and August. Regular subscription rate: $\$ 200.00$ a year (10 issues). Special rate: $\$ 100.00$ a year to individual members of supporting institutions.

Subscriptions, orders for numbers issued in the last three calendar years, and changes of address should be sent to Pacific Journal of Mathematics, P.O. Box 969, Carmel Valley, CA 93924, U.S.A. Old back numbers obtainable from Kraus Periodicals Co., Route 100, Millwood, NY 10546.

The Pacific Journal of Mathematics at P.O. Box 969, Carmel Valley, CA 93924 (ISSN 0030-8730) is published monthly except for July and August. Second-class postage paid at Carmel Valley, California 93924, and additional mailing offices. Postmaster: send address changes to Pacific Journal of Mathematics, P.O. Box 969, Carmel Valley, CA 93924.

PUBLISHED BY PACIFIC JOURNAL OF MATHEMATICS, A NON-PROFIT CORPORATION

This publication was typeset using $\mathcal{A} \mathcal{S} S-\mathrm{T}_{\mathrm{E}} \mathrm{X}$, the American Mathematical Society's $\mathrm{T}_{\mathrm{E}} \mathrm{X}$ macro system.

Copyright (c) 1993 by Pacific Journal of Mathematics 


\section{PACIFIC JOURNAL OF MATHEMATICS}

Volume $158 \quad$ No. $2 \quad$ April 1993

On the extension of Lipschitz functions from boundaries of subvarieties to

201 strongly pseudoconvex domains

K. ADACHI and HiRoshi KAJIMOTO

On a nonlinear equation related to the geometry of the diffeomorphism group 223

DaVid DaI-WAi BaO, JACQUES LAFONTAINE and TUdOR S. RATIU

Fixed points of boundary-preserving maps of surfaces

ROBERT F. BROWN and BRIAN SANDERSON

On orthomorphisms between von Neumann preduals and a problem of Araki 265

L. J. BUNCE and JOHN DAVID MAITLAND WRIGHT

Primitive subalgebras of complex Lie algebras. I. Primitive subalgebras of 273 the classical complex Lie algebras

\section{V. CHEKALOV}

$L^{n}$ solutions of the stationary and nonstationary Navier-Stokes equations in 293 $R^{n}$

\section{ZHI MiN CHEN}

Some applications of Bell's theorem to weakly pseudoconvex domains

XiAO JUN HUANG

On isotropic submanifolds and evolution of quasicaustics

STANISŁAW JANECZKO

Currents, metrics and Moishezon manifolds

SHANYU JI

Stationary surfaces in Minkowski spaces. I. A representation formula

JIANGFAN LI

The dual pair $(U(1), U(1))$ over a $p$-adic field

Courtney Hughes Moen

Any knot complement covers at most one knot complement 